\title{
Deutscher Bibliotheksverband begrüßt Gesetz zur Sonntagsöffnung für Öffentliche Bibliotheken in NRW und setzt sich weiterhin für Bundesregelung ein
}

http://doi.org/10.1515/bd-2020-0003

Der Deutsche Bibliotheksverband e.V. (dbv) begrüßt das Gesetz zur Sonntagsöffnung von Öffentlichen Bibliotheken in Nordrhein-Westfalen vom 9. Oktober 2019. Anders als wissenschaftlichen Bibliotheken, Museen und Theatern war es Öffentlichen Bibliotheken nicht gestattet, sonntags zu öffnen. Nordrhein-Westfalen hat nun landesweit die Möglichkeit dazu geschaffen. Der dbv setzt sich seit vielen Jahren dafür ein, eine entsprechende Regelung im Bundesarbeitszeitgesetz so zu erweitern, dass eine Sonntagsöffnung bundesweit auch Öffentlichen Bibliotheken ermöglicht wird.

Dazu der Bundesvorsitzende des Deutschen Bibliotheksverbandes Prof. Dr. Andreas Degkwitz: „Die Ermöglichung der Sonntagsöffnung in Deutschlands bevölkerungsreichstem Bundesland durch die Änderung der Bedarfsgewerbeverordnung bedeutet eine große Chance für die Öffentlichen Bibliotheken und bietet die Grundlage für eine verstärkte Nutzung. Kommunalen Bibliotheken in NRW wird es nun gestattet, für Familien, Berufstätige und andere Zielgruppen, die während der Woche wenig Zeit haben, attraktive Programme anzubieten. In Zeiten, in denen gesellschaftlicher Zusammenhalt besonders nötig ist, geht von diesem Gesetz ein wichtiges Signal aus“.

Der dbv setzt sich bereits seit vielen Jahren für eine bundeseinheitliche Regelung für die Sonntagsöffnung von Öffentlichen Bibliotheken ein. „Das Bundesarbeitszeitgesetz muss so geändert werden, dass es in allen Bundesländern möglich wird, Fachpersonal auch sonntags einzusetzen und Bibliotheken zu öffnen“, so Degkwitz weiter. „Wir fordern daher den Gesetzgeber auf, diese Gesetzesänderung zügig umzusetzen. Wichtig ist jedoch, gleichzeitig für eine ausreichende Personal- und Finanzausstattung in den Bibliotheken zu sorgen, ohne die eine Umsetzung nicht erfolgreich erfolgen kann.“

Nähere Informationen zu dem Thema unter https://www.bibliotheksverband. $\mathrm{de} / \mathrm{dbv} /$ themen/sonntagsoeffnung.html. 


\section{Der Deutsche Bibliotheksverband e.V. (dbv)}

Der Deutsche Bibliotheksverband e.V. (dbv) vertritt mit seinen mehr als 2.100 Mitgliedern bundesweit rund 10.000 Bibliotheken mit 25.000 Beschäftigten und 11 Mio. Nutzer^innen. Sein zentrales Anliegen ist es, Bibliotheken zu stärken, damit sie allen Bürger^innen freien Zugang zu Informationen ermöglichen. Der Verband setzt sich ein für die Entwicklung innovativer Bibliotheksleistungen für Wissenschaft und Gesellschaft. Als politische Interessensvertretung unterstützt der dbv die Bibliotheken, insbesondere auf den Feldern Informationskompetenz und Medienbildung, Leseförderung und bei der Ermöglichung kultureller und gesellschaftlicher Teilhabe für alle Bürger`innen.

\section{Kontakt:}

Deutscher Bibliotheksverband e.V. (dbv)

Kristin Bäßler, Leitung Kommunikation / Pressesprecherin

Tel.: 030 / 6449899 25, E-Mail: presse@bibliotheksverband.de

URL: www.bibliotheksverband.de 